EPJ Web of Conferences 59, 01013 (2013)

DOI: $10.1051 /$ epjconf/20135901013

(C) Owned by the authors, published by EDP Sciences, 2013

\title{
Experiments in ICF, materials science, and astrophysics
}

\author{
Bruce A. Remington \\ Lawrence Livermore National Laboratory, NIF Directorate, Livermore, CA 94550, USA
}

\begin{abstract}
We have been developing HED experiments on high power ICF lasers over the past two decades that span (1) the radiative hydrodynamics of ICF capsule physics; (2) the high pressure, high strain rate, solid-state dynamics relevant to novel concepts for ICF and hypervelocity impacts in space and on Earth; and (3) the shock driven turbulence of exploding stars (supernovae). These different regimes are separated by many orders of magnitude in length, time, and temperature, yet there are common threads that run through all of these phenomena, such as the occurrence of hydrodynamic instabilities. Examples from each of these three seemingly very disparate regimes are given, and the common theme of hydrodynamic instability evolution is explored.
\end{abstract}

\section{INTRODUCTION}

We describe experiments, starting in about 1990 on the Nova laser, transitioning to the Omega laser in 2000, and starting up on NIF in 2010, to study the Rayleigh-Taylor (RT) instability in ICF, solid-state plastic flow, and in astrophysics, in particular, supernova ( $\mathrm{SN}$ ) explosions. The experiments focused on ablation front RT instability in ICF are described in Sec. 2; RT evolution in solid-state plastic flow is described in Sec. 3, and RT dynamics in SN explosions is discussed in Sec. 4. We conclude with comments about extending these types of experiments onto NIF in Sec. 5.

\section{RAYLEIGH-TAYLOR INSTABILITY IN INERTIAL CONFINEMENT FUSION}

In the period of 1990-2000, we carried out an extensive series of RT experiments done in indirect drive using a shaped radiation drive, as part of the ICF research program on the Nova laser. In Fig. 1a, we show the measured planar ablation front RT dispersion curve from radiatively driven $\mathrm{CH}(\mathrm{Br})$ foils [1-3] vs. classical RT growth at an embedded, $\mathrm{CH}(\mathrm{Br})$ - Ti interface [4]. The ablation front RT growth vs. perturbation wavelength (lower, red curve) displays strong ablative stabilization, with the shortest wavelength ripples $(\lambda=20 \mu \mathrm{m})$ hardly growing at all. In contrast, the embedded interface RT growth (blue curve) shows the highest growth at the shortest wavelengths $(\lambda=10 \mu \mathrm{m}$ and $20 \mu \mathrm{m})$, using the same drive and diagnostic. This represents an experimental demonstration of the effect of ablative RT stabilization, which is an essential effect for the success of ICF ignition.

In Fig. 1b, we examined the effect of spherical convergence on RT growth, by experimentally comparing single mode RT growth in an ablatively driven planar foil, compared to a spherically converging capsule, again using the same drive and diagnostic [5,6]. The converging case (red curve) shows greater RT growth for a given distance accelerated, s (see inset of Fig. 1b). When the RT growth is plotted as a function of distance accelerated divided by ripple wavelength, $(\mathrm{s} / \lambda)^{1 / 2}$, however, this difference disappears, and the two cases evolve identically. Up to a convergence of $\sim 2$ in the linear regime, the only difference between planar and spherical is the shrinking wavelength in the convergent case, since the linear regime classical RT growth rate goes as $\gamma_{R T} \sim(\mathrm{s} / \lambda)^{1 / 2}$.

This is an Open Access article distributed under the terms of the Creative Commons Attribution License 2.0, which permits unrestricted use, distribution, and reproduction in any medium, provided the original work is properly cited. 

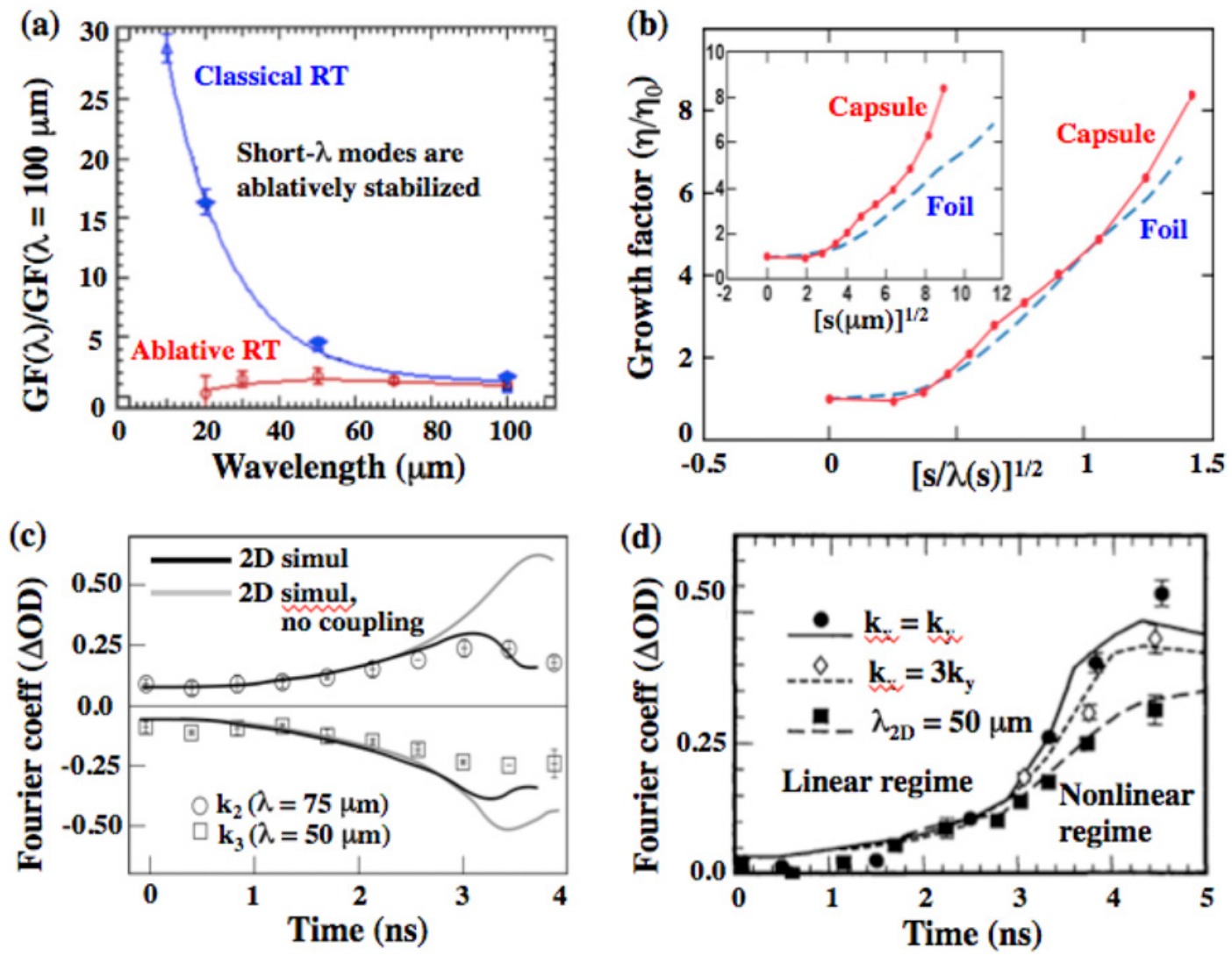

Figure 1. (a) Experimental Rayleigh-Taylor (RT) dispersion curves for ablation-front vs embedded interface (classical) growth [4]. (b) Ablation front RT growth for planar $\mathrm{CH}(\mathrm{Br})$ foils vs. spherical $\mathrm{CH}(\mathrm{Ge})$ capsules, demonstrating the effect of convergence on single mode growth [5]. (c) Experiments of RT growth factors vs. time for a 2-mode sinusoidal pattern corresponding to superposition of wavelengths of $\lambda_{3}=50$ and $\lambda_{2}=75 \mu \mathrm{m}$. The smooth curves represent simulations for the 2-mode superposed pattern, including nonlinear mode coupling (black) and for each wavelength simulated alone (gray curves). The difference represents the perturbation strength being shifted into the coupled terms, $\mathrm{k}_{3} \pm \mathrm{k}_{2}[3,7]$. (d) Experiments and simulations of 2D vs. 3D single-mode perturbations growing at the ablation front of an $\mathrm{x}$-ray driven $\mathrm{CH}(\mathrm{Br})$ foil. The three curves and symbols correspond to $\mathrm{a} \mathrm{k}_{\mathrm{x}}=\mathrm{k}_{\mathrm{y}}$ square (egg crate pattern) $3 \mathrm{D}$ mode, $\mathrm{a} \mathrm{k}_{\mathrm{x}}=3 \mathrm{k}_{\mathrm{y}}$ stretched $3 \mathrm{D}$ mode, and the corresponding $2 \mathrm{D}$ mode, $\mathrm{k}_{\mathrm{x}}=2 \pi / \lambda_{2 \mathrm{D}}, \mathrm{k}_{\mathrm{y}}=0$, all with the same magnitude wave vector $\left(\mathrm{k}_{\mathrm{x}}^{2}+\mathrm{k}_{\mathrm{y}}^{2}\right)^{1 / 2}[8,9]$.

In Fig. 1c, we show the results from 2D planar mode-coupling experiments [3, 7]. We show the RT growth factor vs. time for a 2-mode pattern corresponding to the superposition of $\lambda_{2}=75 \mu \mathrm{m}$ and $\lambda_{3}=50 \mu \mathrm{m}$. In the linear regime, the individual modal components grow identically to their expected growth had this been a single mode experiment. Once the nonlinear regime is entered, mode coupling commences, and Fourier strength is redistributed out of the two pre-existing modes, here, $\mathrm{k}_{3}$ and $\mathrm{k}_{2}$, where $\mathrm{k}=2 \pi / \lambda$, spawning the new coupled models $\mathrm{k}_{3} \pm \mathrm{k}_{2}$ at shorter and longer wavelengths. This mode coupling process in Fourier space marks the onset of bubble merger in physical space, as this Fourier space coupling broadens the dominant bubbles and sharpens the dominant spikes.

Finally, we show in Fig. 1d the results of ablation front RT experiments comparing 2D vs. 3D single-mode growth $[8,9]$. This work was motivated by the emergence of 3D simulations indicating a difference in the RT growth in the nonlinear regime between 2D and 3D [10, 11]. A single model, 3D square "egg crate" pattern $\left(\mathrm{k}_{\mathrm{x}}=\mathrm{k}_{\mathrm{y}}\right)$, where $\lambda_{\mathrm{x}}=\lambda_{\mathrm{y}}=71 \mu \mathrm{m}$, is compared to the equivalent $2 \mathrm{D}$ 
("tin roof") case, with the same magnitude wave vector $\mathrm{k}$, where $\lambda_{2 \mathrm{D}}=50 \mu \mathrm{m}$. The growth vs. time of the Fourier modes for each case are shown in Fig. 1d, as well as with the stretched or elongated egg crate pattern, $\mathrm{k}_{\mathrm{x}}=3 \mathrm{k}_{\mathrm{y}}$ case. The smooth curves correspond to $3 \mathrm{D}$ simulations with the radiationhydrodynamics code HYDRA. In the linear regime, all three patterns grow identically, since they each have the same magnitude wave number, $\mathrm{k}=\left(\mathrm{k}_{\mathrm{x}}^{2}+\mathrm{k}_{\mathrm{y}}^{2}\right)^{1 / 2}$. In the nonlinear regime, however, the 3D $\mathrm{k}_{\mathrm{x}}=\mathrm{k}_{\mathrm{y}}$ bubble starts to grow the fastest, with the 2D bubble growing the least. Based on potential flow theory [12], the asymptotic bubble velocity in the $2 \mathrm{D}$ case is $\mathrm{v}_{2 \mathrm{D}} \sim 0.23(\mathrm{~g} \lambda)^{1 / 2}$, for Atwood number $\mathrm{A}=1$ (which is a reasonable approximation for ablation-front RT growth). The corresponding $3 \mathrm{D}$ case, however, is $\mathrm{V}_{3 \mathrm{D}} \sim 0.36(\mathrm{~g} \lambda)^{1 / 2}$, which is $50 \%$ higher. In the nonlinear regime, $3 \mathrm{D}$ symmetric bubbles grow the fastest. Together with a series of RT instability experiments in cylindrically converging geometry $[13,14]$, this completed our work on indirect drive, ablation front RT growth, as part of the Nova Technical Contract $[15,16]$.

\section{RAYLEIGH-TAYLOR INSTABILITY IN SOLID-STATE PLASTIC FLOW}

We next describe a new regime of science developed on high power ICF lasers over the past 15 years: solid-state plastic flow at very high pressures and strain rates. We developed the ability to force RT growth to occur in a metal foil, where the metal foil remained in the solid state, allowing us to study plastic flow at high pressures $\left(\mathrm{P}_{\max } \sim 1 \mathrm{Mbar}\right)$ and very high strain rates $\left(\mathrm{d} \varepsilon / \mathrm{dt} \sim 10^{7} \mathrm{~s}^{-1}\right)$. This was modelled after the high explosives driven, $\mathrm{P}_{\max } \sim 100 \mathrm{kbar}, \mathrm{d} \varepsilon / \mathrm{dt} \sim 10^{5} \mathrm{~s}^{-1}$ experiments of Barnes et al. [17] only at 10-100x higher pressures and strain rates. To be able to interpret our experimental results required the development of a new solid-state strength model, namely, the multiscale model, to treat this new regime of plastic flow [18]. This work is summarized, for a small subset of our results, in Fig. 2 [19-25]. The basic mechanism of solid-state plastic flow is very different from fluid flow in the plasma state, as illustrated schematically in Fig. 2a [26]. At the microscopic level, deformation occurs via dislocation generation and transport. A dislocation is a break at the lattice level of magnitude one lattice spacing. When a large density of dislocations, $\rho_{\text {disloc }} \sim 10^{9}-10^{12} \mathrm{~cm}^{2}$, is transported, macroscopic deformation (strain, $\varepsilon$ ) results. The basic relation that describes this is Orowan's equation, $\mathrm{d} \varepsilon / \mathrm{dt} \sim$ $\rho_{\text {disloc }} \mathrm{v}_{\text {disloc }} \mathrm{b}$, where $\mathrm{v}_{\text {disloc }}$ and $\mathrm{b}$ correspond to average dislocation velocity and Burgers vector, namely, the lattice level step size, as the dislocation glides along its slip plane. In the thermal activation regime, pre-existing dislocations are pinned against barriers, and get freed by thermal fluctuations, after which they glide to the next barrier, where they get stopped. In the phonon drag regime, the applied shear stresses are large enough that dislocations glide above all barriers, and are resisted by scattering phonons in the lattice, which generate a drag.

One of our experiments studied RT instability induced solid-state plastic flow at $\sim 1$ Mbar pressures and very high strain rates, $10^{6}-10^{8} \mathrm{~s}^{-1}$. We show the results of a series of RT experiments in vanadium (V), a bcc metal of moderate density, $\rho_{0} \sim 6.1 \mathrm{~g} / \mathrm{cm}^{3}$. The method for generating the ramped, quasiisentropic drive that keeps the metal sample well below melt, uses a 2-step process [19, 27, 28]. A strong shock is launched across a plastic "reservoir", that unloads across a vacuum gap and stagnates on the sample to be studied. The ram pressure of the incoming unloading plasma transforms to thermal pressure when this unloading reservoir stagnates on the sample. The resulting pressure vs. time is illustrated in Fig. $2 b$ and the simulated sample temperature vs. predicted melt temperature is shown in the inset. The sample had an imposed ripple prepared in it, protected from the heat from the stagnating reservoir by a thin polyethylene $\left(\mathrm{CH}_{2}\right)$ foil or "heat shield". This ramp loaded pressure causes the sample to accelerate. The accelerating rippled sample is Rayleigh-Taylor unstable, and the rippled amplitude grows with time, moderated by the material strength.

In Fig. 2c, we show the result of this RT growth in the solid-state V sample at the pressures and temperatures shown in Fig. 2b [21, 22]. The solid red symbols show the experimental growth factor vs. time. The result of $2 \mathrm{D}$ simulations in a radiation-hydrodynamics code, assuming no strength in the samples, is shown by the top curve. The results of simulations using the Steinberg-Guinan and PTW 

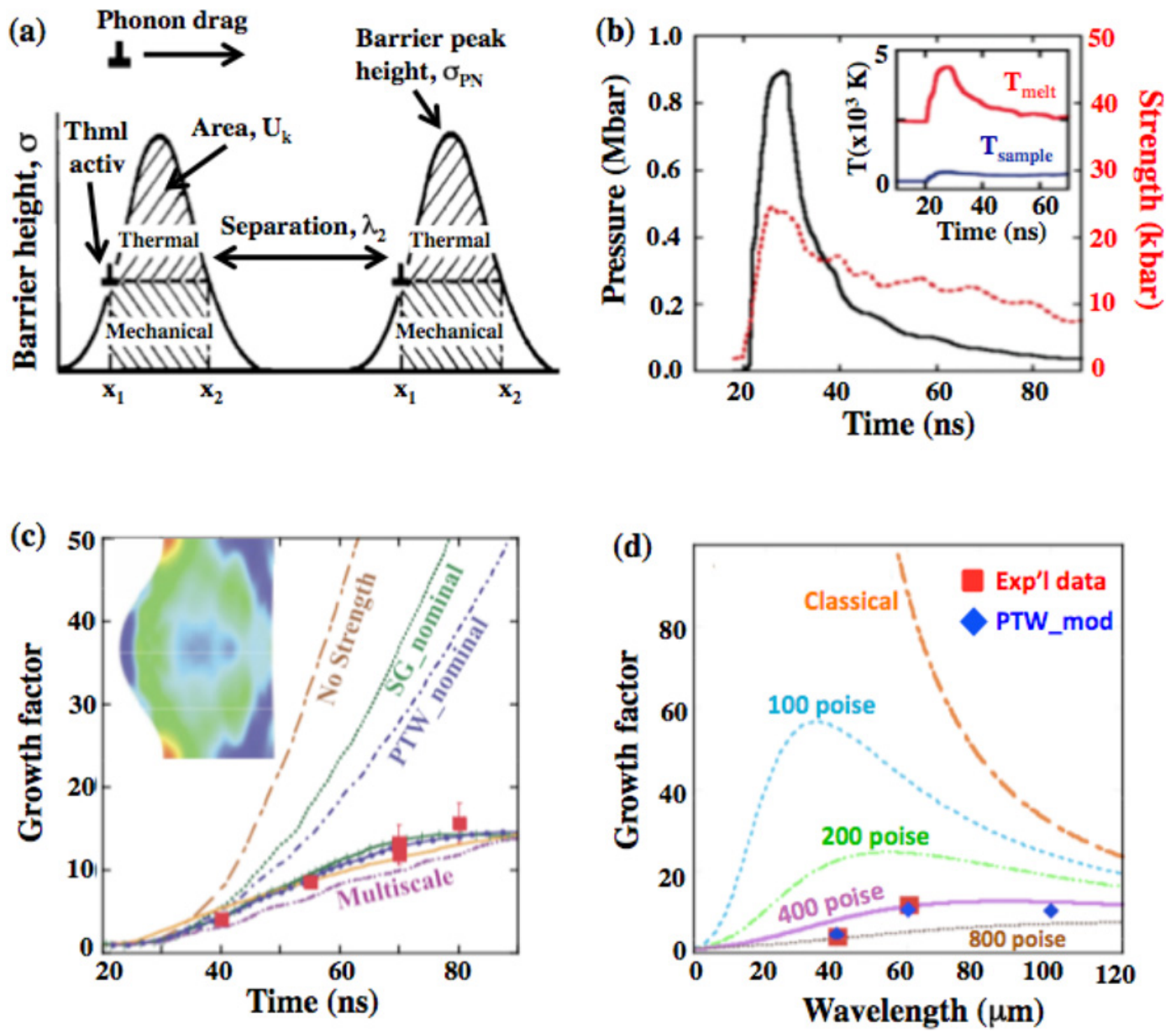

Figure 2. (a) Cartoon of edge dislocations pinned against a barrier in the thermal activation regime vs. dislocations gliding over the tops of the barriers in the phonon drag regime [26]. (b) Experiments developed to drive solidstate Rayleigh-Taylor (RT) plastic flow in sputtered samples of vanadium (V). Simulated pressure and flow stress (strength) vs. time for the V sample. The inset shows the simulated V temperature vs. its expected melt temperature vs. time [21, 22]. (c) Experimental measurements of RT growth factor, shown as the solid square plotting symbols vs. time. The smooth curves correspond to $2 \mathrm{D}$ simulations using no strength (top curve), the Steinberg-Guinan (S-G) strength model (second curve from the top), PTW strength model (third curve from the top), and multiscale strength model (bottom smooth curve). The second from the bottom curve is a calculation of linear regime RT growth in a viscous fluid, where the viscosity was used as a fitting parameter. The remaining two curves going essentially through the data points correspond to the S-G and PTW models, where parameters have been adjusted to reproduce the experimental results [22]. (d) Solid-state RT dispersion curves created as growth factor vs. perturbation wavelength for three different wavelengths $(40,60,100 \mu \mathrm{m})$, versus the results from the RT dispersion curve at a series of increasing viscosities (lowest at the top) [21, 22].

strength models are shown as the next two curves from the top; both simulations over-predict the RT growth, meaning their predicted strengths at these conditions were too low. Parameter adjustments can be made in both models that then bring the simulations into agreement with the experimental data. The lowest curve corresponds to a simulation using the LLNL multiscale strength model [18], which comes much closer to the experimental data. The conclusion from the multiscale simulations for these conditions of $\sim 1 \mathrm{Mbar}, 10^{7} \mathrm{~s}^{-1}$ pressures and strain rates in vanadium is that the deformation occurs in 
the phonon drag regime (see Fig. 2a). When we query the simulation, this corresponds to a peak average flow stress (strength) of $\sim 25 \mathrm{kbar}$, as shown in Fig. $2 \mathrm{~b}$.

If we assume that the flow stress at these high plastic strain rates can be approximated as an effective lattice viscosity, then using the RT dispersion curve for viscous flow, we can fit the data, as shown in Fig. 2d. The best fit corresponds to an effective viscosity of $\sim 400$ Poise [21, 22]. Writing down an approximate equation relating viscosity with flow stress, $\sigma \sim\left(6^{1 / 2}\right)(\mathrm{d} \varepsilon / \mathrm{dt})(\mu)$ [29], we can estimate a strength of 20-30 kbar, assuming a strain rate of $\mathrm{d} \varepsilon / \mathrm{dt} \sim(1 / 3)\left(\mathrm{d} \rho_{\text {mat }} / \mathrm{dt}\right) / \delta \mathrm{t} \sim 2-3 \times 10^{7} \mathrm{~s}^{-1}$. One can further relate the effective lattice viscosity to the phonon drag coefficient, via $\mu / \mathrm{B} \sim \mathrm{M}^{2} /\left(\rho_{\text {disloc }} \mathrm{b}^{2}\right)$, where $\mathrm{M} \sim 2.75$ is the Taylor factor, and $\mathrm{B}$ is the phonon drag coefficient. If we assume $\rho_{\text {disloc }} \sim$ $10^{11} \mathrm{~cm}^{-2}$ from recovery experiments [24] or from dislocation dynamics simulations [18], then using $\mu \sim 400$ Poise gives $\mathrm{B} \sim 10^{-3} \mathrm{~Pa} \cdot \mathrm{s}=10^{-2} \mathrm{dyn} \cdot \mathrm{s} / \mathrm{cm}^{2}$ for the phonon drag coefficient.

\section{RAYLEIGH-TAYLOR INSTABILITY IN SUPERNOVA EXPLOSIONS}

We next turn to core-collapse supernovae ( $\mathrm{SNe}$ ) [30-35], and describe the development of scaled experiments to study $\mathrm{SN}$ explosion dynamics in the laboratory. In Fig. 3a, we show an example of a 2D core-collapse SN simulation of SN1987A [33]. Once a massive star burns up to iron in its core, there is no longer net energy gain by the fusion reactions, and the core thermal pressures can no longer balance the inwardly directed force of gravity; the core collapses. The collapse continues until the core reaches the density of nuclear degenerate matter, at which point the degeneracy pressure stops the collapse, and an enormously strong rebound shock is launched that blows the star apart. As the shock traverses each layer of decreasing density as it exits the star, it triggers RM followed by RT instability growth. The result of all this instability growth is that core material from the star is ejected to the surface much sooner and at much higher velocities than expected, based on simulations such as those shown in Fig. 3a. This problem is very relevant to how the heavy elements (heavier than $\mathrm{Fe}$ ) are ejected into interstellar space, where they ultimately reappear in subsequent star and planet formation. Given the deeply nonlinear, (and in reality turbulent) flows shown in Fig. 3a, one wonders how reliable such simulations are. Hence, our effort to develop scaled dynamic experiments to rigorously test these codes and simulations in localized regions of space and time.

We show in Fig. 3b the interface velocities vs. time from the first scaled SN experiments [36, 37]. The experiments were done on the Nova laser, using a 1-ns duration x-ray drive to launch a strong shock through an $85 \mu \mathrm{m}$ thick planar $\mathrm{Cu}$ layer mated to a $500 \mu \mathrm{m}$ thick $\mathrm{CH}_{2}$ plastic layer. The interface had a pre-imposed sinusoidal perturbation of $200 \mu \mathrm{m}$ wavelength with a $20 \mu \mathrm{m}$ amplitude machined into it. Once the shock passes the interface, a rarefaction follows, and the shock accelerated interface then experiences a protracted deceleration. There is first Richtmyer-Meshkov (RM) followed by an extended period of Rayleigh-Taylor (RT) growth. The overall perturbation growth was measured by side-on radiography, with an example raw radiograph shown in the inset of Fig. 3c. The evolution of the RT spike front and bubble front data vs. time are shown in the Fig. 3c, along with the simulations from CALE (a Lab code) and PROMETHEUS (a SN code used in astrophysics). The experimental RT bubble and spike envelopes are reproduced well with the simulations; detailed perturbation shapes, however, from the two codes differ in detail from each other and from the data. In the nonlinear (but not yet turbulent) regime, the codes reproduce the overall perturbation (peak-to-valley) size, at least for single mode perturbations, but the detailed shapes are suspect. There is considerable sensitivity in the detailed shapes to the EOS used.

To address scalability, we examine the Euler equations. Provided one holds the Euler number $\mathrm{Eu}=(\mathrm{h} / \tau) /(\mathrm{P} / \rho)^{1 / 2}$ fixed, then pressure $(\mathrm{p})$, density $(\rho)$, spatial scale $(\mathrm{h})$, and time scale $(\tau)$ can be varied across many orders of magnitude, and the equations (and physics) remain invariant to this scale transformation. For example, if the characteristic spatial scale, density, and pressure for the two systems (star and laboratory) are related by $\mathrm{x}_{\mathrm{s}}=\mathrm{ax}_{l}, \rho_{\mathrm{s}}=\mathrm{b} \rho_{l}, \mathrm{p}_{\mathrm{s}}=\mathrm{cp}_{l}$, then the Euler equations remain invariant under this scale transformation, provided the time scales in the laboratory experiment obey 
(a)

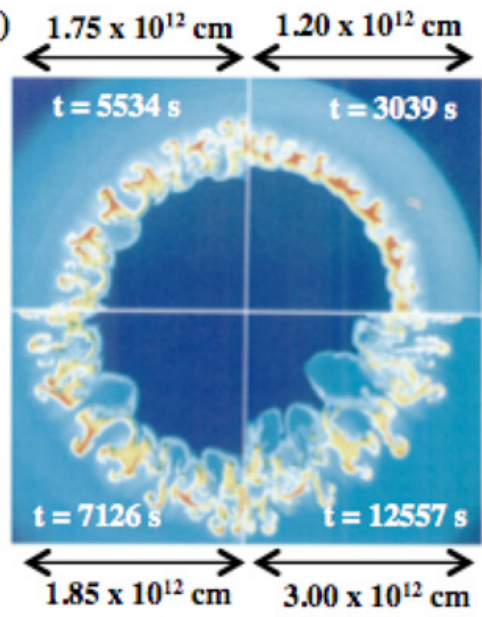

(c)

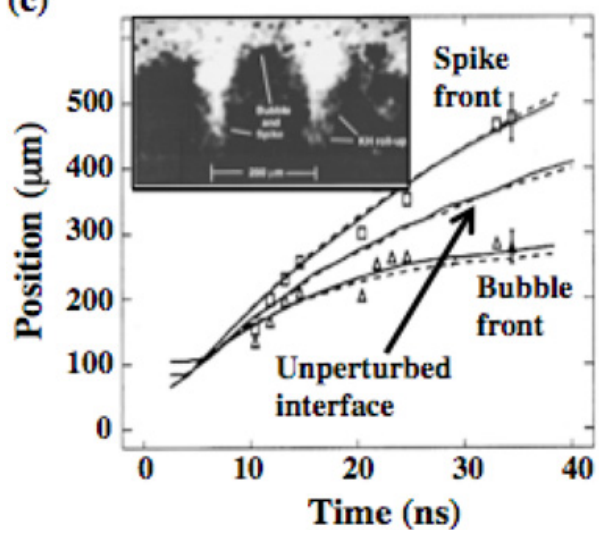

(b) Laser experiment time (nanoseconds)

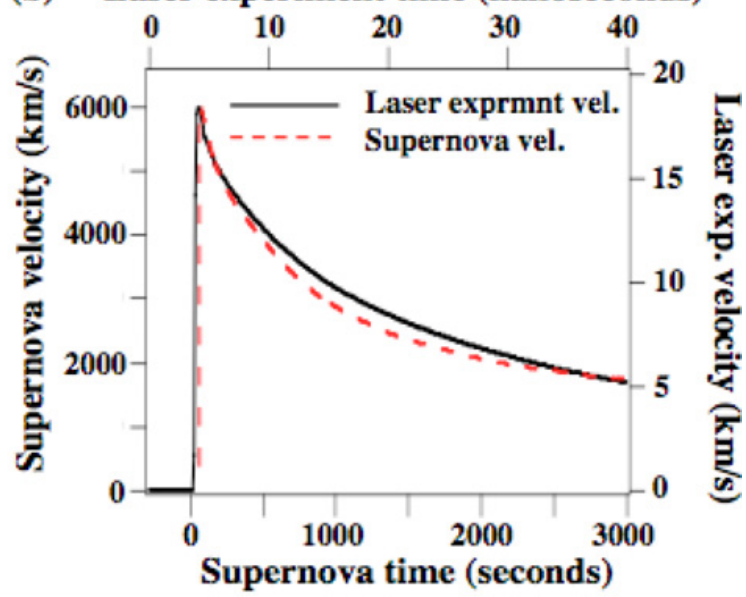

(d)

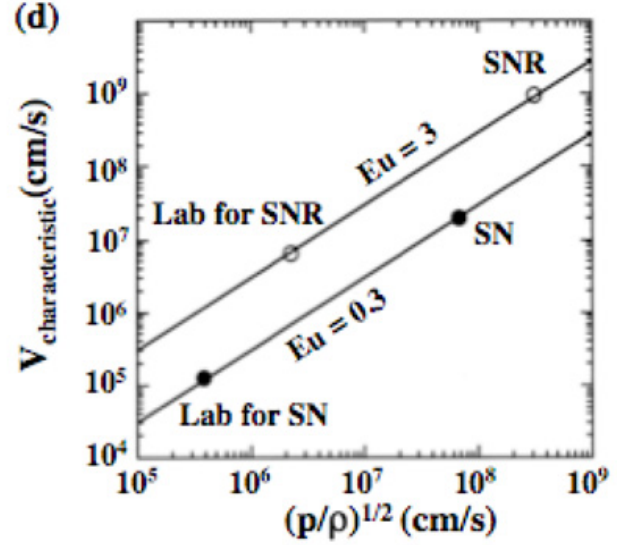

Figure 3. (a) A 2D simulation of SN1987A, a Type-II, core-collapse supernova, assuming a multimode perturbation. The color corresponds to contours of partial density (total density times mass fraction) of ${ }^{4} \mathrm{He}$ at four different times from the simulation, spanning $\sim 3000 \mathrm{sec}$ to $\sim 12000 \mathrm{sec}$, with the spatial extents indicated by the double arrows [33]. (b) Results from the first scaled supernova hydrodynamics experiment, done on the Nova laser [36, 37]. The curves show the interface velocity vs. time for the He-H interface in SN1987A, and the scaled laser experiment. (c) Scaled Rayleigh-Taylor experiments done on the Nova laser to test supernova explosion hydrodynamics codes in 2D hydrodynamic instability experiments. The data are compared with a 2D simulation using the laboratory code CALE and a tabular EOS, and the astrophysics code PROMETHEUS with an ideal EOS [36]. The inset shows an in-flight x-ray radiograph of this first scaled supernova experiment done on the Nova laser $[36,37]$. (d) Euler scaling shows how the experiments are related to the supernova dynamics by means of the Euler scale transformation [38].

$t_{s}=a(b / c)^{1 / 2} t_{l}$. Provided the Euler number is the same, the physics does not know the difference whether it is describing the SN explosion hydrodynamics at spatial and time scales of $10^{11}-10^{12} \mathrm{~cm}$ and $10^{3}-10^{4} \mathrm{~s}$ or that of the scaled laboratory experiment at spatial and time scales of $10-100 \mu \mathrm{m}$ and 10-100 ns. This Euler scaling is illustrated graphically in Fig. 3d. These scaled SN experiments have been moved to the Omega laser over the past 10 years and generated a wealth of scaled dynamics to compare SN simulation codes against [39-43]. A variety of interesting additional novel HED laboratory astrophysics applications, as well as $\mathrm{SNe}$, are described in several review articles [44-46]. 


\section{CONCLUSION AND FUTURE DIRECTIONS}

In ICF, we are now developing experiments on NIF to assess and reduce the impact of hydrodynamic instabilities on ignition experiments [47]. We are starting the solid-state plastic flow experiments to study material strength to NIF to reach much higher pressures [48]. We are also developing next generation supernova hydrodynamics experiments on NIF to push the RT evolution into the turbulent regime, and assess how radiation affects the RT evolution [49].

\section{References}

[1] B.A. Remington et al., Phys. Fluids B 4, 967 (1992)

[2] B.A. Remington et al., Phys. Fluids B 5, 2589 (1993)

[3] B.A. Remington et al., Phys. Plasmas 2, 241 (1995)

[4] K.S. Budil et al., Phys. Rev. Lett. 76, 4536 (1996)

[5] C. Cherfils et al., Phys. Rev. Lett. 83, 5507 (1999)

[6] S.G. Glendinning et al., Phys. Plasmas 7, 2033 (2000)

[7] B.A. Remington et al., Phys. Rev. Lett. 73, 545 (1994)

[8] M.M. Marinak et al, Phys. Rev. Lett. 75, 3677 (1995)

[9] E.M. Campbell et al., Laser Part. Beams 15, 607 (1997)

[10] D. Shvarts et al., Phys. Plasmas 2, 2465 (1995)

[11] J. Hecht et al., Laser Particle Beams 13, 423 (1995)

[12] D. Layzer, Astrophys. J. 122, 1 (1955)

[13] W.W. Hsing and N.M. Hoffman, PRL 78, 3876 (1997)

[14] W.W. Hsing et al., Phys. Plasmas 4, 1832 (1997)

[15] J.D. Lindl, Phys. Plasmas 2, 3933 (1995)

[16] J.D. Lindl et al., Phys. Plasmas 11, 339 (2004)

[17] J.F. Barnes et al., J. Appl. Phys. 45, 727 (1974)

[18] N.R. Barton et al., J. Appl. Phys. 109, 073501 (2011)

[19] B.A. Remington et al., Metallurgical and Materials Transactions A 35A, 2587 (2004)

[20] B.A. Remington et al., Material Science and Technology 22, 474 (2006)

[21] H-S. Park et al., Phys. Rev. Lett. 104, 135504 (2010)

[22] H-S. Park et al., Phys. Plasmas 17, 056314 (2010)

[23] R.E. Rudd et al., MRS Bull. 35, 999 (2010)

[24] M.A. Meyers et al., Acta Mat. 51, 1211 (2003)

[25] M.A. Meyers et al., J. of Materials (JOM) 62, 24 (2010)

[26] M.A. Meyers, Dynamic Behavior of Materials, (John Wiley \& Sons, New York, NY, 1994)

[27] J. Edwards et al., Phys. Rev. Lett. 92, 075002 (2004)

[28] K.T. Lorenz et al., High En. Den. Phys. 2, 113 (2006)

[29] J.D. Colvin et al., J. Appl. Phys. 93, 5287 (2003)

[30] W.D. Arnett, Astrophys. J. 319, 136 (1987)

[31] W.D. Arnett, Astrophys. J. 331, 377 (1988)

[32] W.D. Arnett et al., Astrophys. J. 341, L63 (1989)

[33] E. Muller, B. Fryxell, and W.D. Arnett, Astron. Astrophys. 251, 505 (1991)

[34] K. Kifonidis et al., AA. 408, 621 (2003)

[35] K. Kifonidis et al., AA. 453, 661 (2006)

[36] J. Kane et al., Astrophys. J. 478, L75 (1997)

[37] B.A. Remington et al., Phys. Plasmas 4, 1994 (1997)

[38] D. Ryutov et al., Astrophys. J. 518, 821 (1999)

[39] H.F. Robey et al., Phys. Plasmas 8, 2446 (2001)

[40] A. Miles et al., Phys. Plasmas 12, 056317 (2005) 
[41] C.C. Kuranz et al., Astrophysics and Space Science 298, 9 (2005)

[42] C.C. Kuranz et al., Astrophysics and Space Science 307, 115 (2007)

[43] C.C. Kuranz et al., Phys. Plasmas 16, 056310 (2009)

[44] B.A. Remington, R.P. Drake, and D.D. Ryutov, Rev. Mod. Phys. 78, 755 (2006)

[45] M.D. Rosen, PoP 3, 1803 (1996)

[46] R.P. Drake, J. Geophys. Res. 104, 14505 (1999)

[47] B.A. Hammel et al., Phys. Plasmas 18, 056310 (2011)

[48] S.T. Prisbrey et al., Phys. Plasmas 19, 056311 (2012)

[49] C.C. Kuranz et al., Astrophysics and Space Science 322, 49 (2011) 